\title{
Patch-based local Turbulence Compensation in anisoplanatic Conditions
}

\author{
Adam W.M. van Eekeren", Maarten C. Kruithof, Klamer Schutte, Judith Dijk, \\ Miranda van Iersel, Piet B.W. Schwering \\ TNO, PO Box 96864, 2509 JG The Hague, The Netherlands
}

\begin{abstract}
Infrared imagery over long ranges is hampered by atmospheric turbulence effects, leading to spatial resolutions worse than expected by a diffraction limited sensor system. This diminishes the recognition range and it is therefore important to compensate visual degradation due to atmospheric turbulence. The amount of turbulence is spatially varying due to anisoplanatic conditions, while the isoplanatic angle varies with atmospheric conditions. But also the amount of turbulence varies significantly in time. In this paper a method is proposed that performs turbulence compensation using a patch-based approach. In each patch the turbulence is considered to be approximately spatially and temporally constant. Our method utilizes multi-frame super-resolution, which incorporates local registration, fusion and deconvolution of the data and also can increase the resolution. This makes our method especially suited to use under anisoplanatic conditions. In our paper we show that our method is capable of compensating the effects of mild to strong turbulence conditions.
\end{abstract}

Keywords: Turbulence compensation, deconvolution, image restoration, super-resolution

\section{INTRODUCTION}

Atmospheric turbulence effects deteriorate imagery which is captured over long ground-to-ground ranges. This diminishes the recognition range and it is therefore important to compensate visual degradation due to atmospheric turbulence. Turbulence compensation is a challenging problem because 1) the amount of turbulence is spatially varying due to anisoplanatic conditions while the isoplanatic angle, i.e. the angle over which the turbulence can be assumed constant, varies with atmospheric conditions and 2) the amount of turbulence varies significantly in time.

According to Roggeman and Welsh [1,2] there are three different approaches to compensate for turbulence effects in images: 1) adaptive optics techniques, 2) post-processing techniques and 3) a combination of both techniques. The first approach is often used within astronomy applications. Except for space telescopes, all high-end telescopes are hindered by the Earth's atmosphere. Modern telescopes typically include an adaptive optics part, which corrects the atmospheric distortion. A typical assumption in such a system is that the turbulence effect is constant over the field of view of the telescope. For astronomy applications it is often assumed that the turbulence is caused by one or two relatively thin atmospheric layers, in effect resulting in one or two phase screens. In anisoplantic conditions in astronomy multiconjugate adaptive optics techniques are under development, using multiple guide stars. However, for ground-to-ground applications the full imaging path will be much closer to the ground and will be within a single atmospheric layer. This will result in a more complex turbulence setup. In effect, the isoplanatic angle will often be much smaller than the field of view of the camera system. This means that a closed loop adaptive optics system such as used in an astronomy application does not work for ground-to-ground imaging.

The second approach is a software-based approach where the images are processed (preferably in real-time) after recording. There exist many different algorithms which can be divided into two categories: 1) deconvolution methods [3] and 2) lucky imaging methods [4]. Of course there are also methods that use a combination of techniques.

The third approach to compensate for the effects of turbulence uses hardware (wavefront modulator) as well as software. In this approach a wavefront modulator is placed in front of the optics (in free-running mode) to modulate the incoming wavefront with several known modulations. These known modulations are used in post-processing to reconstruct the

*adam.vaneekeren@tno.nl; phone +31 8886 64047; www.tno.nl 
original, not-deteriorated, signal [5,6]. A disadvantage of this approach is that the modulations must be generated really fast and therefore expensive hardware (wavefront modulator) is needed. Also much processing power is required.

In literature many software post-processing techniques are described, such as the methods described in [7, 8] which performs first a global registration followed by deblurring. There also exist methods that perform local registration to cope with anisoplanatic conditions $[9,10]$. Comparable to the latter paper, we developed a post-processing technique based on multi-frame super-resolution reconstruction. Such an approach incorporates registration, temporal image fusion and deblurring. Our proposed method copes with anisoplanatic conditions by using local registration. The major differences with the approach of [10] are that we use frame selection, a robust approach for registration and that our registration is less computational complex.

In respectively Section 2 and 3 the assumed imaging model and the proposed method are described. In Section 4 results of our method on different datasets are depicted. Finally, conclusions will be drawn in Section 5.

\section{IMAGING MODEL}

This section describes the assumed imaging model. The camera's field-of-view, the scene, is modeled as a properly sampled 2D HR image $\mathbf{z}$ (see Figure 1). It is assumed that this HR image is free of degradation to motion, blur and noise. The output frames $\mathbf{y}_{k}$ are a result of the effects caused by atmospheric turbulence and the camera itself on the scene.

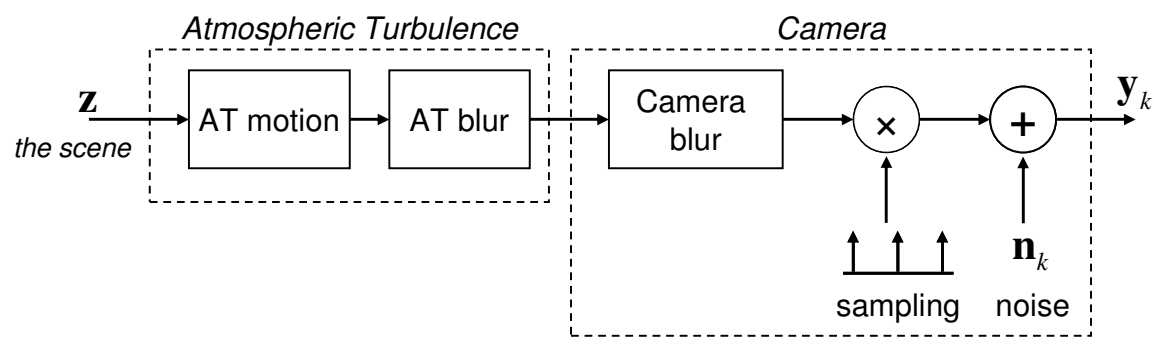

Figure 1. Flow diagram illustrating the degradation of a properly sampled scene $\mathbf{Z}$ into a turbulence and camera deteriorated image $\mathbf{y}_{k}$.

The degradation of camera frame $\mathbf{y}_{k}$ can be split up in two parts: a part that is due to the atmospheric turbulence and a part which is caused by the camera.

\subsection{Atmospheric turbulence}

Atmospheric turbulence causes blurring, transformation (motion) and scintillation in the observed image of the scene. All these phenomena are assumed to be constant in a small region (patch) within the field-of-view. The size of such a region is determined by the isoplanatic angle, which is from a theoretical perspective, for a horizontal path through the atmosphere, often smaller than even a pixel. The blurring caused by turbulence is modeled by a time- and spatially varying Gaussian function with standard deviation $\sigma_{t}(x, y, t)$. The transformation which is caused by turbulence is locally modeled by a translation. In this paper scintillation, the effect of illuminance variation, is not modeled because it is not much present in the processed data.

\subsection{Camera}

The camera is modeled by a 'camera blur' (optics and sensor), a sampling device and additive Gaussian distributed noise. The camera blur is modeled by a Gaussian function with standard deviation $\sigma_{\mathrm{c}}$. In this paper it is assumed that the camera blur is small compared to the turbulence blur. Therefore the overall blur is assumed to be a Gaussian with standard deviation $\sigma_{\mathrm{tot}} \approx \sigma_{\mathrm{t}}$. 
The imaging model as depicted in Figure 1 can be described by the following equation:

$$
\mathbf{y}_{k}=\mathbf{R} \mathbf{W}_{k} \mathbf{M}_{k} \mathbf{z}+\mathbf{n}_{k}
$$

where $\mathbf{M}_{k}$ represents the transformation matrix, $\mathbf{W}_{k}$ describes the blurring matrix and $\mathbf{R}$ is the resampling matrix, which is typically the identity matrix when no resolution enhancement takes place.

\section{PROPOSED METHOD}

This section describes the proposed method for compensating the effects of turbulence in images. An overview of this method is depicted in Figure 2. First a reference image is calculated from the first $N_{0}$ frames, then the next frames are selected, registered (first globally, then locally) and deblurred resulting in a turbulence-compensated frame $\mathbf{z}_{k}$.

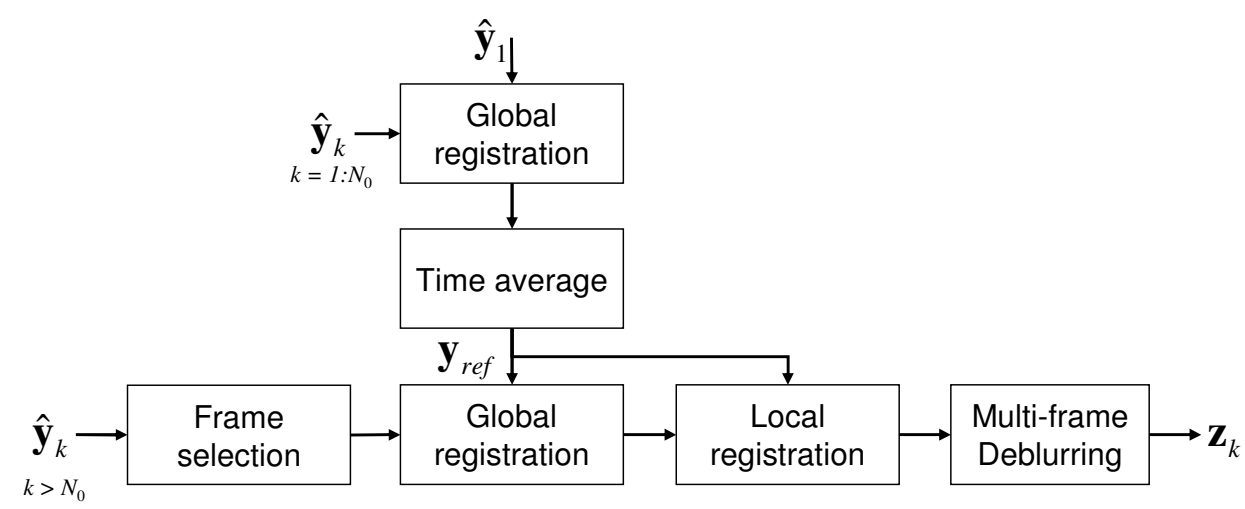

Figure 2. Flow diagram of the proposed method for compensation of the effects of atmospheric turbulence in images.

\subsection{Reference frame}

To perform registration a reference image is necessary. Such a reference image $\left(\mathbf{y}_{\text {ref }}\right)$ is calculated using global registration, assuming a translational motion model, of the first $N_{0}$ frames of an image sequence followed by time averaging those registered frames. Considering the anisoplanatic conditions of turbulence the reference image will be blurry. However, the global structure of the scene is stabilized and serves as a good reference. A typical range for setting $N_{0}=[20 . .50]$ using frame rates between 30 and $70 \mathrm{~Hz}$.

\subsection{Frame selection}

The first step of the pipeline of the proposed method is frame selection. This is done by calculating the $99^{\text {th }}$ percentile of the Gaussian gradient magnitude $(\sigma=4)$ of each of $N_{t}$ frames and selecting the $N_{s}$ frames with the highest percentiles. This results in an output sequence which is less fluctuating in sharpness compared to an output sequence without frame selection. Typical parameter setting ranges are $N_{t}=[20 . .75]$ and $N_{s}=[10 . .50]$ for frame rates between 30 and $70 \mathrm{~Hz}$.

\subsection{Registration}

The second step consists of registration. First the frames $\left(k>N_{0}\right)$ are globally registered against the reference image using a gradient-based shift estimator [11] and then locally using optical flow [12]. Optical flow estimates a per pixel local translation. To speed up computation of the optical flow first local translations are calculated at a sub-sampled and equally spaced grid in the image. From this grid the optical flow is interpolated. Typical parameter settings used are a grid space of 10 pixels and a window size of $8 \times 8$ pixels. 


\subsection{Deblurring}

The third step consists of multi-frame deblurring, which utilizes the multi-frame super-resolution method of Hardie et al. $[13,14]$. The main difference is that our method uses optical flow registration while Hardie uses global translation as registration. Equal to Hardie the blurring is assumed spatially and temporally constant. To estimate the scene at frame $k$ $\left(\mathbf{z}_{k}\right)$, the following cost function is minimized:

$$
C\left(\mathbf{z}_{k}\right)=\sum_{m}^{N_{s}}\left(\hat{\mathbf{y}}_{m}-\mathbf{R} \mathbf{W}_{k} \mathbf{M}_{k} \mathbf{z}_{k}\right)^{2}+\lambda_{r} \sum_{n}\left(L\left(\mathbf{z}_{k}\right)\right)^{2},
$$

where $\hat{\mathbf{y}}_{m}$ are $N_{s}$ observed frames used for deblurring, $\mathbf{M}_{k}$ represents the transformation matrix containing the global and local registration, $\mathbf{W}_{k}$ describes the blurring matrix and $\mathbf{R}$ is the resampling matrix. The final part of the cost function is the so-called regularization part which forces a smoothness constraint on the solution. Here $\lambda_{r}$ is a weighting factor and $L$ indicates a Laplacian operator. The cost function is minimized using a conjugate-gradient optimization. Typical parameter setting ranges for the deblurring part of our method are: resampling factor $=[1 . .4]$, blurring $\sigma_{\text {tot }}=$ [1..3], $\lambda_{r}=\left[10^{-3} . .10^{-1}\right]$ and [5..10] iterations for the conjugate-gradient minimization procedure.

Note that the proposed method is implemented in MATLAB and is not optimized for speed. Processing a batch of $N_{s}=$ 10 frames of $256 \times 256$ pixels takes about 17 seconds. An optimized implementation is likely to run in real-time.

\section{RESULTS}

To test the performance of the proposed method a few datasets, deteriorated by turbulence, are processed.

\subsection{FATMOSE dataset}

This data is captured during the FATMOSE (False Bay ATMOspheric Experiment) trial organized by TNO and the Institute of Marine Technology (IMT) in False Bay, South Africa in 2009 - 2010. In this experiment images are captured with a Marlin F-033B camera looking at a beach station at the other side of the False Bay at a distance of $15.7 \mathrm{~km}$. The specifications of the camera are depicted in Table 4.1. More information about the FATMOSE trail can be found in [15].

Table 4.1. Specifications of Marlin F-033B camera and the used optics.

\begin{tabular}{|l|l|}
\hline Max. responsivity & $\lambda=800 \mathrm{~nm}$ \\
\hline Image size & $640 \times 480$ pixels \\
\hline Pixel size & $9.9 \times 9.9 \mu \mathrm{m}$ \\
\hline Shutter time, frame rate & $100 \mu \mathrm{s}, 30 \mathrm{~Hz}$ \\
\hline Focal length & $2030 \mathrm{~mm}$ \\
\hline IFOV & $5 \times 5 \mu \mathrm{rad}$ \\
\hline
\end{tabular}

The data which is processed was captured at 20 April 2010 at $16.35 \mathrm{~h}$ under weak turbulence conditions. The refractive index structure parameter, $C_{n}^{2}$, indicates the strength of the turbulence and was measured at that time with the BLS as $C_{n}^{2}=2.9 \times 10^{-16} \mathrm{~m}^{-2 / 3}$. Following theory this means that the captured imagery at the range of $15.7 \mathrm{~km}$ is totally anisoplanatic. According to Fried [16] the isoplanatic angle for a homogeneous path can be expressed as:

$$
\theta_{0}=0.95\left(\frac{2 \pi}{\lambda}\right)^{-6 / 5} L^{-8 / 5}\left(C_{n}^{2}\right)^{-3 / 5}
$$


with $\lambda$ the wavelength and $L$ the path length. Given the parameters of the used data $\left(\lambda=800 \mathrm{~nm}, L=15.7 \mathrm{~km}, C_{n}^{2}=\right.$ $2.9 \times 10^{-16} \mathrm{~m}^{-2 / 3}$ ), this leads to an isoplanatic angle of $\theta_{0}=2.1 \mu \mathrm{rad}$, which is smaller than the IFOV of the camera. According to this angle it is necessary to process the data using patches smaller than one pixel, which in practice is not possible. However, it gives us a clue that the effects of turbulence are very local and the patches must not be too big. In this paper a patch size of $8 \times 8$ pixels is used. An example of a deteriorated input frame together with a high-resolution close-up of the beach station is depicted in Figure 3.

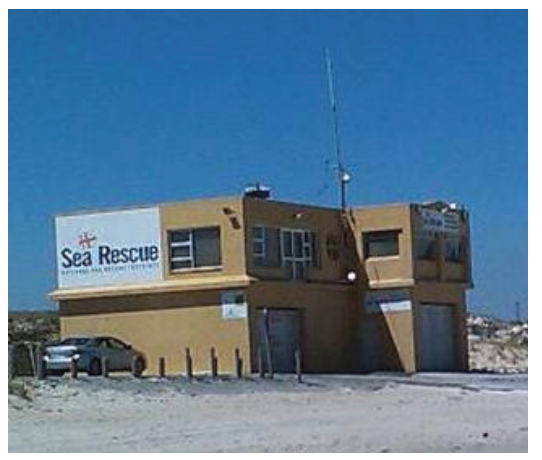

(a)

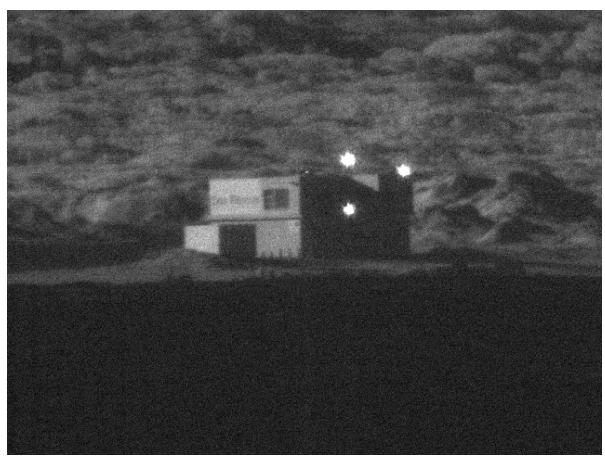

(b)

Figure 3. (a) High-resolution visual close-up of the beach station, (b) near infrared image taken at $15.7 \mathrm{~km}$ deteriorated by turbulence of the same beach station, taken at 20 April 2010 at $16.35 \mathrm{~h}$.

A sequence of 100 deteriorated frames is processed with the method described in the previous section. The following parameter settings are chosen experimentally: $N_{0}=20$ frames, $N_{t}=20$ frames, $N_{s}=10$ frames, resampling factor 1 , blurring $\sigma_{\text {tot }}=1, \lambda_{r}=0.1$ and 5 iterations for the minimization procedure. Input frame 75 together with the processed frame is depicted in Figure 4. It is clear that the processed frame is sharper and contains less noise. The stabilizing effect of our method however can only be appreciated by watching the resulting video.

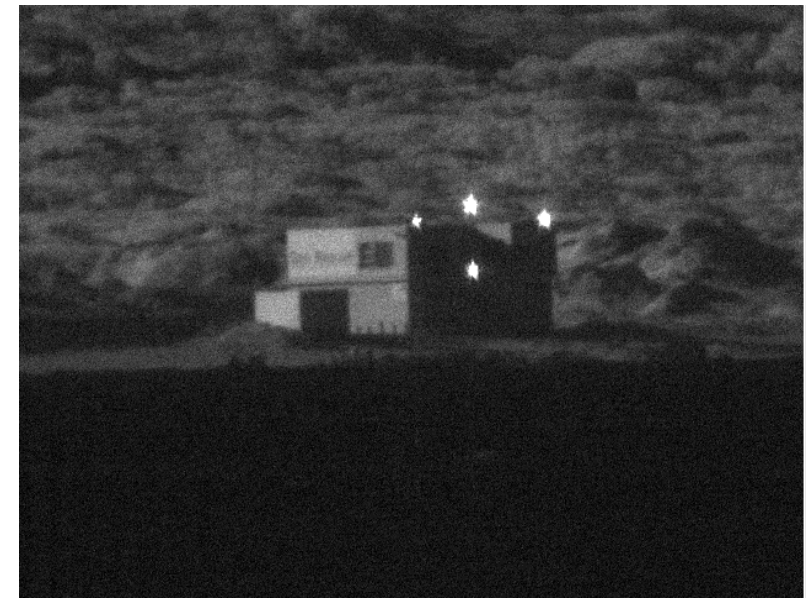

(a)

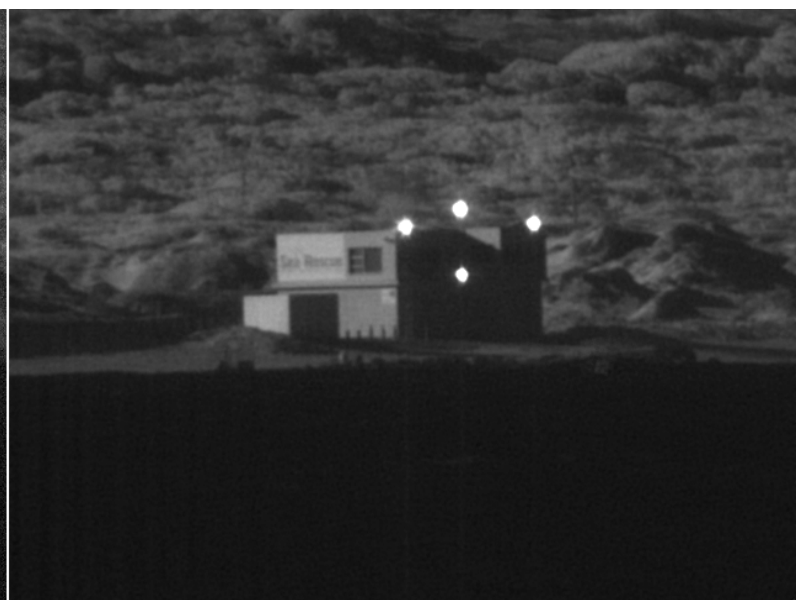

(b)

Figure 4. (a) Deteriorated input frame 75 taken at 20 April 2010 at 16.35h, (b) Processed frame with the proposed method.

A second experiment was performed on the same dataset to demonstrate the resolution enhancement capabilities of the proposed method. For this experiment a region-of-interest (front of building) of 150 frames is processed. The following parameter settings are used: $N_{0}=50$ frames, $N_{t}=75$ frames, $N_{s}=50$ frames, resampling factor 1 and 4 , blurring $\sigma_{\text {tot }}=1$, 
$\lambda_{r}=0.01$ and 0.1 respectively and 5 iterations for the minimization procedure. The results after processing at frame 100 are shown together with the corresponding input frame in Figure 5. In the processed frames the words "Sea Rescue" are much better visible, especially after resolution enhancement with factor 4 .

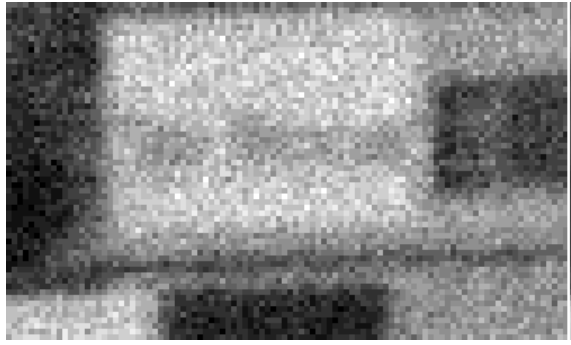

(a)

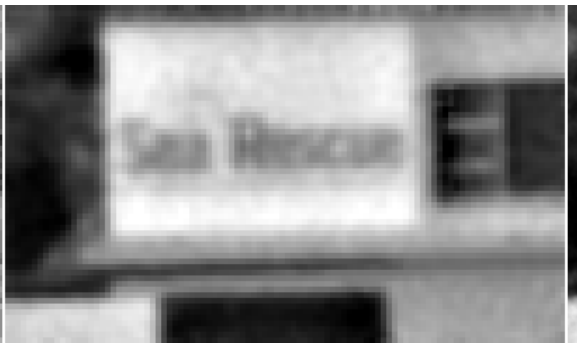

(b)

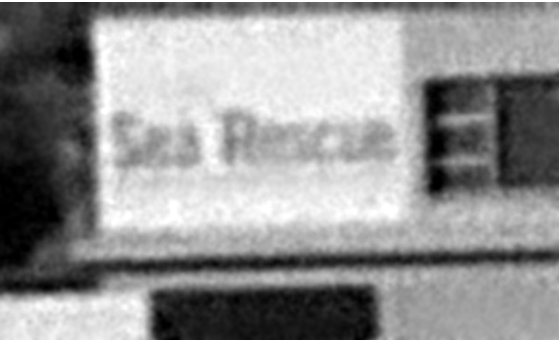

(c)

Figure 5. (a) Region-of-interest of deteriorated input frame 100, (b) Resulting frame after processing with resampling factor 1, (c) Resulting frame after processing with resampling factor 4. On both (b) and (c) local contrast enhancement is applied [17] for better visualisation.

\subsection{Dayton dataset}

The Dayton dataset is captured during a NATO SET-165 trial in Dayton, USA, beginning of October 2011 [18]. Two different image sequences are processed. The first sequence is captured at 12 October 2011 at $15.35 \mathrm{~h}$ and contains a building with the word "NATO" attached to it. The distance over which the imagery is captured, is approximately $7 \mathrm{~km}$. The refractive index structure parameter was measured at that time as $C_{n}^{2}=6.9 \times 10^{-16} \mathrm{~m}^{-2 / 3}$. The camera used is the AVT Stingray F-080B with a maximum frame size of $1032 \times 778$ pixels and a pixel depth of 14-bit monochrome. For the optics a Celestron C8 is used. The "NATO" sequence is captured with a resolution of $1032 \times 264$ pixels, a frame rate of $69 \mathrm{~Hz}$, a shutter time of $25 \mathrm{~ms}$ and a camera gain of $16.2 \mathrm{~dB}$.

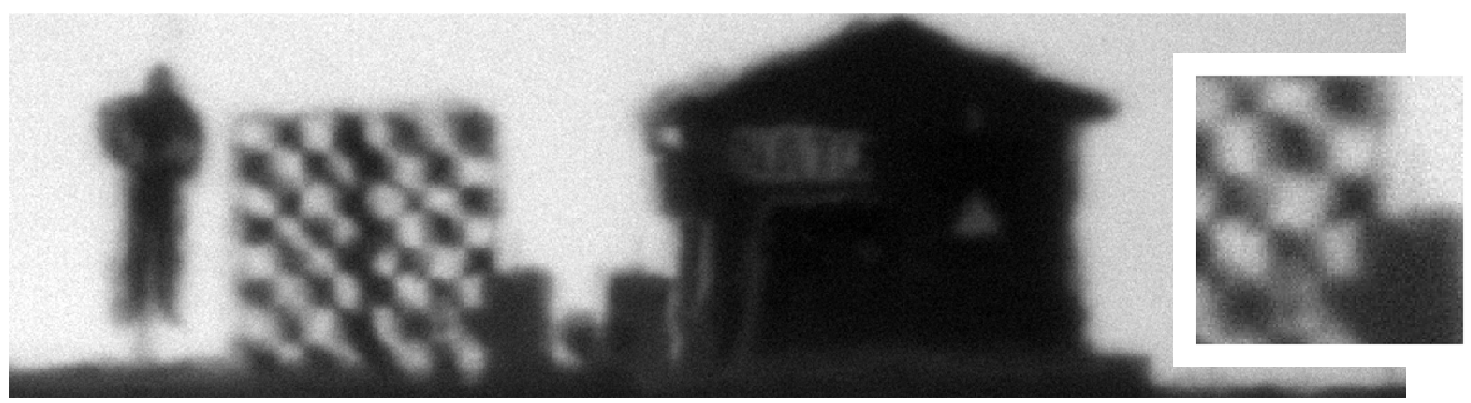

(a)

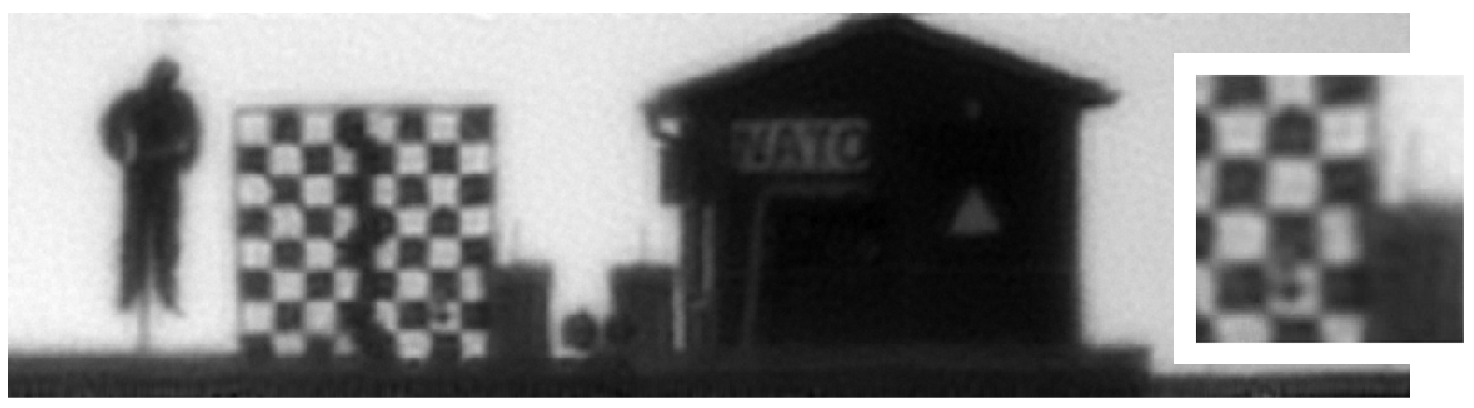

(b)

Figure 6. (a) One of the deteriorated frames of the "NATO" sequence taken on 12 October 2011 at $15.35 \mathrm{~h}$ with on the right side a detail of the checkerboard. (b) Corresponding processed frame by the proposed method. 
The following parameter settings were used to process 200 frames: $N_{0}=20$ frames, $N_{t}=20$ frames, $N_{s}=10$ frames, resampling factor 1 , blurring $\sigma_{\mathrm{tot}}=3, \lambda_{r}=10^{-3}$ and 10 iterations for the minimization procedure. One of the processed frames is shown together with an input frame in Figure 6, although the result can be appreciated best by watching a video. Looking at the images in Figure 6, it is clear that more detail is visible in the processed frame, such as on the checkerboard and the NATO sign on the building.

Another sequence from the Dayton dataset is processed showing an antenna tower. This sequence is captured on 12 October 2011 at $16.15 \mathrm{~h}$ with a resolution of $552 \times 776$ pixels, a frame rate of $31.6 \mathrm{~Hz}$, a shutter time of $8.3 \mathrm{~ms}$ and a camera gain of $22.7 \mathrm{~dB}$. The refractive index structure parameter was measured at that time as $C_{n}^{2}=8.2 \times 10^{-16} \mathrm{~m}^{-2 / 3}$.

The following parameter settings were used to process 200 frames: $N_{0}=20$ frames, $N_{t}=20$ frames, $N_{s}=10$ frames, resampling factor 1 , blurring $\sigma_{\mathrm{tot}}=3, \lambda_{r}=0.1$ and 10 iterations for the minimization procedure. In Figure 7 input frame 100 is shown together with the corresponding processed frame. Here it is very clear that processing helps to visualize more details.

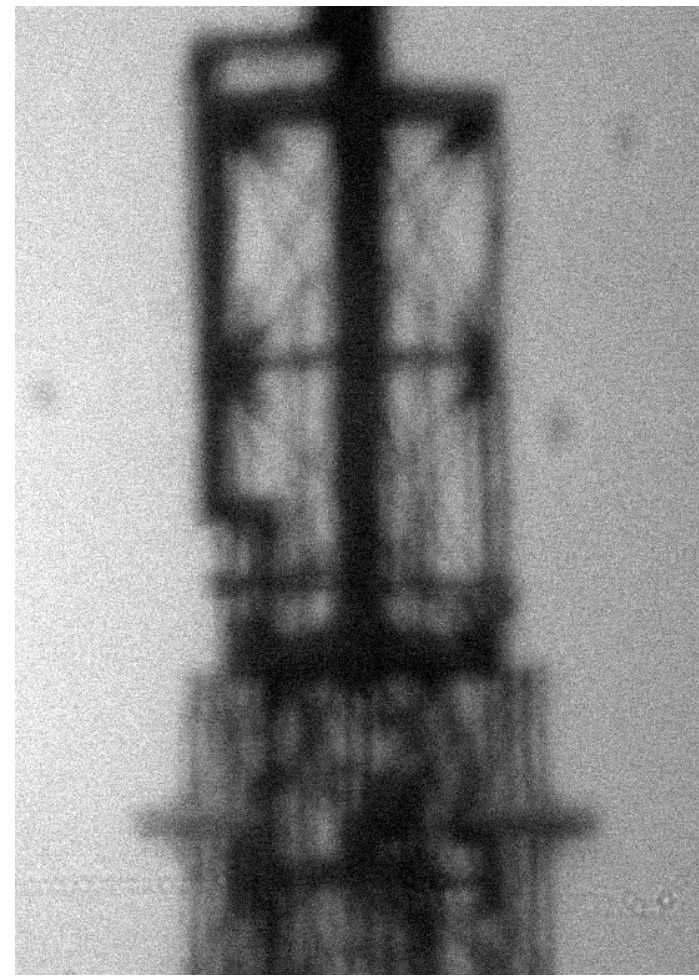

(a)

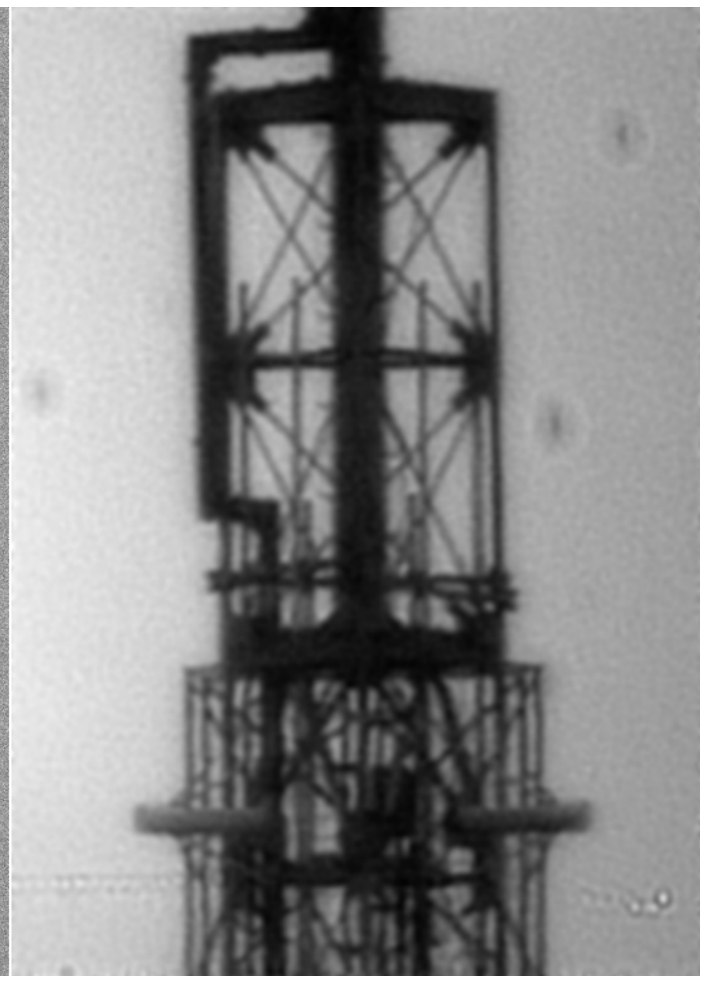

(b)

Figure 7. (a) One of the deteriorated frames of the "Antenna" sequence taken on 12 October 2011 at 16.15h. (b) Corresponding processed frame by the proposed method.

\section{CONCLUSIONS AND FUTURE WORK}

A method is presented to compensate the effects of turbulence in static image sequences. The results show that the proposed method is capable of stabilizing and deblurring such image sequences. Also it is shown that resolution enhancement is possible in images captured during weak to mild turbulence conditions. The presented method enables an operator to see more details at a large distance and it enlarges the recognition range. 
In the presented method the registration is done locally. However, the deblurring is done at a global scale and the amount of deblurring is determined manually. Future work will focus on local blur estimation [19] and performing the deblurring part of our method on a local scale.

\section{ACKNOWLEDGEMENTS}

The authors would like to thank Arie de Jong, Peter Fritz and Koen Benoist for their work in the FATMOSE trial and we would also like to thank the NATO SET-165 group for the availability of the Dayton dataset.

\section{REFERENCES}

[1] Roggeman, M. and Welsh, B., "Imaging through turbulence," CRC Press, Boca Raton, USA (1996).

[2] van Eekeren, A. W. M., Schutte, K., Dijk, J., Schwering, P. B. W., and van Iersel, M., "Turbulence compensation: an overview," Proc. SPIE Infrared Technology and Applications XXXVIII 8353 (2012).

[3] Huebner, C. S. and Greco, M., "Blind deconvolution algorithms for the restoration of atmospherically degraded imagery: a comparative analysis," Proc. SPIE Optics in Atmospheric Propagation and Adaptive Systems XI 7108 (2008).

[4] Fried, D., "Probability of getting a lucky short-exposure image through turbulence," Optical Society of America Journal 68, 1651-1658 (1978).

[5] van Eekeren, A. W. M., Schutte, K., Dijk, J., and Schwering, P. B. W., "Time-varying phase diversity turbulence compensation," Proc. SPIE Infrared Technology and Applications XXXVII 8012 (2011).

[6] van Eekeren, A. W. M., Schutte, K., Dijk, J., and Schwering, P. B. W., "Turbulence compensation using timevarying phase diversity," OPTRO (2012).

[7] Schwering, P. B. W., van den Broek, S. P., and van Iersel, M., "Eo system concepts in the littoral," Proc. SPIE Infrared Technology and Applications XXXIII 6542 (2007).

[8] van Iersel, M. and van Eijk, A. M. J., "Estimating turbulence in images," Proc. SPIE Free-Space Laser Communications X 7814 (2010).

[9] Huebner, C. S., "Compensating image degradation due to atmospheric turbulence in anisoplanatic conditions," Proc. SPIE Mobile Multimedia/Image Processing, Security, and Applications 7351 (2009).

[10] Zhu, X. and Milanfar, P., "Image reconstruction from videos distorted by atmospheric turbulence," Proc. SPIE Visual Information Processing and Communication 7543 (2010).

[11] Pham, T. Q., Bezuijen, M., van Vliet, L. J., Schutte, K., and Hendriks, C. L. L., "Performance of optimal registration estimators," Proc. SPIE Visual Information Processing XIV 5817, 133-144 (2005).

[12] Horn, B. and Schunck, B., "Determining optical flow," Artificial Intelligence 17, 185-203 (1981).

[13] Hardie, R. C., Barnard, K. J., Bognar, J. G., Armstrong, E. E., and Watson, E. A., "High-resolution image reconstruction from a sequence of rotated and translated frames and its application to an infrared imaging system," Optical Engineering 37(1), 247-260 (1998).

[14] van Eekeren, A. W. M., Schutte, K., Oudegeest, O. R., and van Vliet, L. J., "Performance evaluation of superresolution reconstruction methods on real-world data," EURASIP Journal on Advances in Signal Processing , 1-11 (2007).

[15] de Jong, A., Fritz, P., Benoist, K., van Eijk, A., and Schwering, P., "Preliminary results of the fatmose atmospheric propagation trials in the false bay, south africa, november 2009 - july 2010," Proc. SPIE Optics in Atmospheric Propagation and Adaptive Systems XIII 7828 (2010).

[16] Fried, D., “Anisoplanatism in adaptive optics," Optical Society of America Journal 72, 52-61 (1982).

[17] Schutte, K., "Multiscale adaptive gain control of ir images," Proc. SPIE Infrared Technology and Applications XXIII 3061 (1997).

[18] Velluet, M., Vorontsov, M., Espinola, R., Marchi, G., Nicolas, S., Schwering, P., and Riker, J., "Turbulence characterization and image processing data sets from a nato rto set 165 trial in dayton, oh, usa," Proc. SPIE Atmospheric Propagation IX 8380 (2012).

[19] Bouma, H., Dijk, J., and van Eekeren, A., "Precise local blur estimation based on the first-order derivative," Proc. SPIE Visual Information Processing XXI 8399 (2012). 